\title{
Medical Safety
}

$\mathrm{P}$ atient safety in the hospital setting is in infancy. Patient safety in resource constrained setting is in many respects still 'in utero' waiting to be born. In USA, with the mandate by President Bush in 2004, electronic medical records reached center stage with presumption that electronic records could prevent many mishaps. Current adoption rates in inpatient setting are very high and in ambulatory settings are approximately $5-10 \%$ of physician offices but many more are poised to adopt them. Electronic Medical Recording is not the only component in Medical Safety. We in Nepal are quite far from Universal Electronic Medical Recording, but can do a lot in our own systems towards this.

To quote from WHO Patient Safety Curriculum Guide for Medical Students "Health care outcomes have significantly improved with the scientific discoveries of modern medicine. However, studies from a multitude of countries show that with these benefits come significant risks to patient safety. We have learnt that hospitalized patients are at risk of suffering an adverse event. Patients on medication have the risk of medication errors and adverse reactions. A major consequence of this knowledge has been the development of patient safety as a specialized discipline. Clinicians, managers, health-care organizations, governments (worldwide) and consumers must become familiar with patient safety concepts and principles. Everyone is affected. The tasks ahead of health care are immense and require all those involved care to understand the extent of harm to patients and why health care must move to adopt a safety culture. Patient safety education and training is only beginning to occur at all levels. Medical students, as future doctors and health-care leaders, must also be prepared to practice safe health care. Though medical protocols are continuously changing to accommodate the latest discoveries and new knowledge, patient safety knowledge is different from other because it applies to all areas of practice. Clinicians need to know how systems impact on the quality and safety of health care, how poor communication can lead to adverse events and much more. We need to learn how to manage these challenges. Patient safety is not a traditional stand alone discipline; rather, it is one that integrates into all areas of medicine and health care ${ }^{1}$."

The World Health Organization's (WHO) World Alliance for Patient Safety aims to implement patient safety worldwide. Patient safety is everyone's business, all the way from patients to politicians ${ }^{2}$.

\section{Reporting and learning for patient safety}

The most important knowledge in the field of patient safety is how to prevent harm to patients during treatment and care. The fundamental role of patient safety reporting systems is to enhance patient safety by learning from failures of the health care system. Health-care errors are often provoked by weak systems and often have common root causes which can be generalized and corrected. Although each event is unique, there are likely to be similarities and patterns in sources of risk which may otherwise go unnoticed if incidents are not reported and analyzed.

From this issue of Nepal Paediatric Society journal, we are starting a series on Medical safety. This issue will deal with the basics of medical safety. From next issue after giving a relevant background, a case where a medical error (ranging from minor error to near miss) occurred will be briefly described without naming the institution or person. The emphasis will be on improvement of systems, rather than on blaming someone. A system of how to act in such circumstances will be outlined. The process of correction will be described.

\section{References}

1. WHO Patient Safety Curriculum Guide for Medical Schools. 2009.

2. WHO: World Alliance for Patient Safety. Forward Program 2006-2007. 